\title{
Abdominal Emergencies in Patients with Stage IV Melanoma: The Role of Surgery: A Single-centre Experience
}

\author{
DIMITRIOS MANTAS ${ }^{1}$, CHRISTOS DAMASKOS $^{1}$, NIKOLAOS GARMPIS $^{1}$, \\ DIMITRIOS DIMITROULIS ${ }^{1}$, ANNA GARMPI ${ }^{2}$ and HELEN GOGAS ${ }^{3}$
}

\author{
${ }^{1}$ Second Department of Propedeutic Surgery, ${ }^{2}$ Internal Medicine Department, and ${ }^{3}$ First Department of Internal Medicine, \\ Laiko General Hospital, Medical School, National and Kapodistrian University of Athens, Athens, Greece
}

\begin{abstract}
Background/Aim: Metastatic melanoma is an aggressive disease with poor prognosis. Melanoma can potentially involve any organ. In this article, we report on a single-centre experience in emergency surgery for Mlc melanoma. Patients and Methods: Twenty-eight consecutive patients with M1c melanoma underwent surgical exploration due to abdominal emergencies. Pre-operative computed tomography confirmed the diagnosis and the location of the affected site. Pre-operative lactate dehydrogenase serum levels and post-operative histopathology findings were recorded. Results: Intestinal obstruction was the most frequent intraoperative finding (75\%). The ileum was most frequently affected (28.6\%). Multifocal disease and extragastrointestinal tract metastases were present in $25 \%$ of cases each. Lactate dehydrogenase serum level was increased in $75 \%$ of the patients. Most patients underwent an enterectomy. Conclusion: Curative surgery for stage IV melanoma remains debatable, but surgery for patients presenting with abdominal emergencies appears to improve both survival rate and prognosis. Combined novel therapies and surgical resection is currently being studied with promising results.
\end{abstract}

Metastatic melanoma (MM) is an aggressive disease with poor prognosis despite novel chemotherapeutic and immunotherapeutic agents. Advanced melanoma can potentially involve any organ, with the commonest distant sites of metastasis being the skin, lung, and brain. In cases of visceral involvement, MM most frequently involves the liver and the small bowel.

Correspondence to: Dr. Christos Damaskos MD, M.Sc., Ph.D., Second Department of Propedeutic Surgery, Laiko General Hospital, Medical School, National and Kapodistrian University of Athens, 17 Agiou Thoma Street, Athens, 11527, Athens, Greece. Mobile: +306948467790,e-mail: x_damaskos@yahoo.gr

Key Words: Melanoma, metastatic, laparotomy, acute, abdomen, emergency, surgery.
In its latest edition of the cancer staging manual, the American Joint Committee on Cancer (AJCC) has divided patients with systemic disease into three groups: M1a: distant metastasis in skin, soft tissue (including muscle), and/or nonregional lymph nodes; M1b: pulmonary metastasis with/without M1a; M1c: distant metastasis in organs other than the CNS with/without M1a and M1b; and M1d: metastasis in the CNS with/without M1a, M1b, or M1c (1, 2). All groups are further subdivided based on normal or elevated lactate dehydrogenase (LDH) level into group 0 or 1 , respectively (Table I). This classification differs from the previous one and therefore any staging information should be followed by the edition number of the AJCC staging manual used. As expected, patients with M1a disease have the highest survival rate, whilst those with M1c have the lowest, something further supported by existing clinical evidence (3). Recently, the mitotic rate was removed from the list of prognostic factors for MM (1). M1c melanoma carries a median survival of about 6 to 10 months for all age groups $(3,4)$. The vast majority of patients with metastases to the gastrointestinal tract (GI) will not be properly diagnosed until autopsy (5).

Intra-abdominal dissemination of melanoma can produce a variety of atypical findings and symptoms such as weight loss, vague abdominal pain or tenderness, and anaemia. Patients can also present to the Emergency Department with a more dramatic picture of bowel obstruction, intussusception, haemorrhage, or perforation (5). Although an acute abdominal presentation can raise the suspicion of intra-abdominal metastasis in any patient with a history of cutaneous melanoma, diagnosis is definitively reached intra-operatively. In this article, we report on our single-centre experience in emergency surgery for M1c melanoma.

\section{Patients and Methods}

Between January 2009 and December 2017, 28 consecutive patients with M1c melanoma underwent emergency laparotomy by a single senior consultant general surgeon (DM). All patients underwent a diagnostic abdominal computed tomographic (CT) scan preoperatively 
Table I. Classification system of metastatic melanoma according to the latest edition of cancer staging manual of the American Joint Committee on Cancer (8th Edition) (2).

M1 M1a Distant metastasis in skin, soft tissue (including

(1) M1a(0) muscle), and/or non-regional lymph nodes.

M1a(1) Normal LDH

Elevated LDH

M1b Pulmonary metastasis with/without M1a.

M1b(0) Normal LDH

M1b(1) Elevated LDH

M1c Distant metastasis in organs other than

M1c(0) the CNS with/without M1a and M1b.

M1c(1) Normal LDH

Elevated LDH

M1d Metastasis of the CNS with/without M1a, M1b, or M1c. M1d(0): Normal LDH

M1d(1): Elevated LDH

LDH: Lactate dehydrogenase; CNS: central nervous system.

in order to confirm the diagnosis and locate the affected site. All patients were operated on within approximately 4 hours of admission. Patients were Caucasian with a history of MM and had had previous palliative treatment. Their median age was 61 years $($ range $=17-85$ years) and patients were predominantly males $(71.5 \%)$.

Apart from demographic data of the patients, clinical presentation, GI site, primary site, extra-GI metastases, lymph node infiltration, type of surgery, complications, last follow-up, disease-free survival, recurrence and overall survival were recorded. The pre-operative LDH level and histopathology reports were collected for all patients. The 28 patients were followed-up until they died or were lost to follow-up.

\section{Results}

Acute abdominal pain was the main symptom. Intestinal obstruction was the most frequent intraoperative finding (21 cases; $75 \%$ ), followed by intra-abdominal haemorrhage (seven cases; 25\%). The ileum was the most frequently affected part (28.6\%), followed by the jejunum (14.3\%), the rectum $(10.7 \%)$ and the adrenal glands $(7.2 \%)$. Other parts that were affected included the spleen, the stomach, the duodenum and the ileocecal valve in one case for each of these organs. Multifocal disease was present in seven cases (25\%). In seven cases (25\%), extra-GI metastases were present; mainly affecting the omentum. At presentation, the LDH level was increased in 21 out of 28 patients (75\%).

The majority of patients underwent enterectomy. Three patients $(10.7 \%)$ underwent a single enterectomy and nine more $(32.2 \%)$ underwent multiple enterectomies with primary end-to-end enteroenteral anastomoses (ranging from 2-5 resection segments). Other procedures included a right hemicolectomy with primary ileotransverse anastomosis, wedge resection of a gastric lesion, a palliative gastrojejunal bypass procedure for gastrointestinal obstruction in a patient

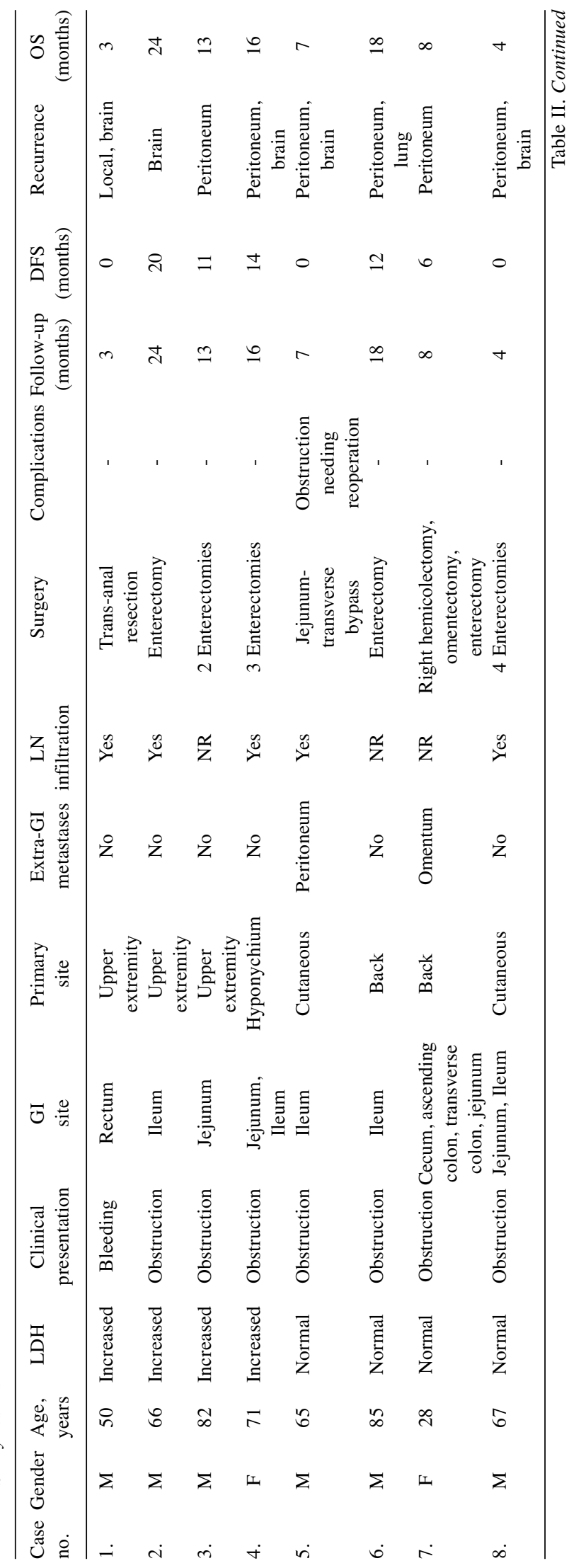




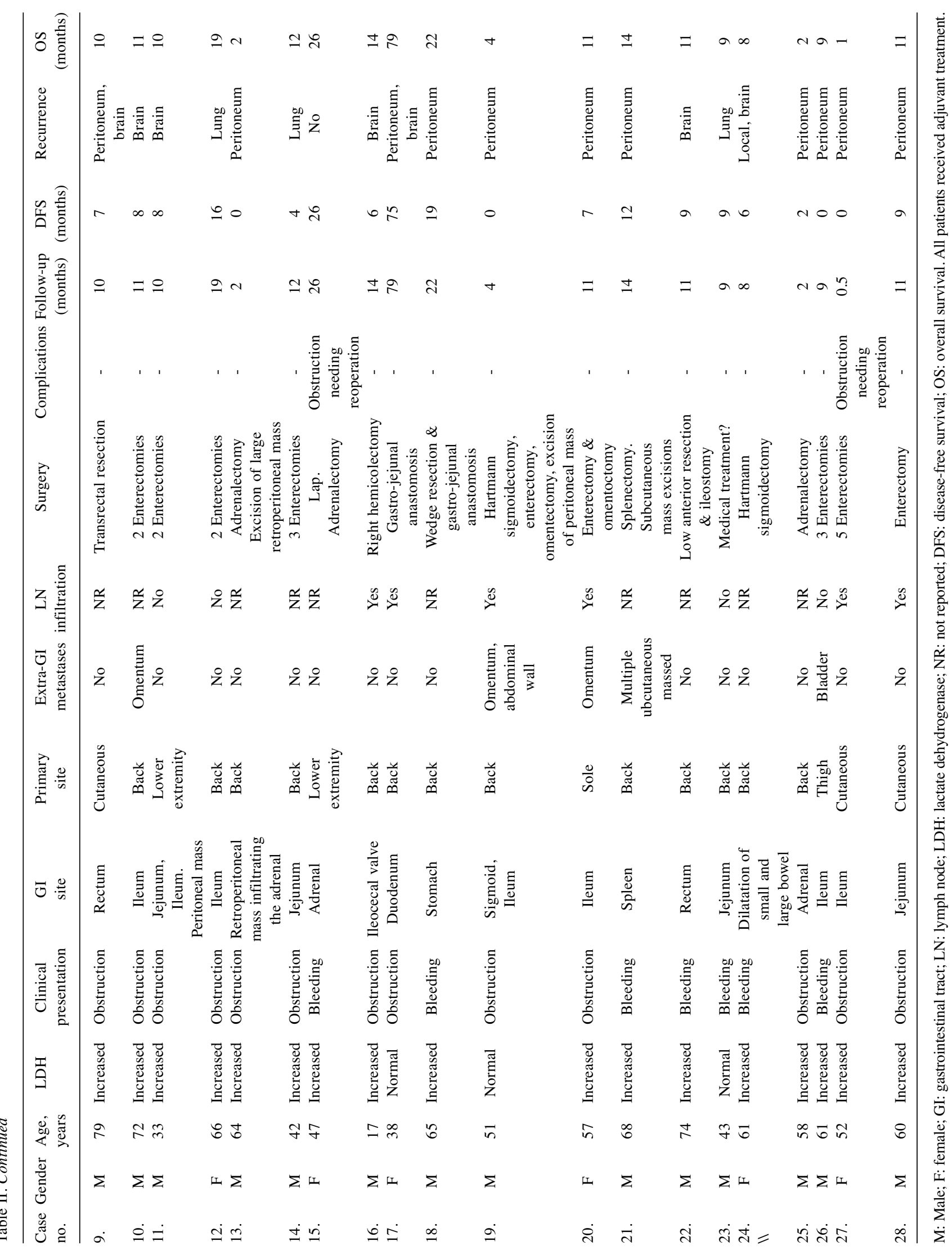


Table III. Immunohistochemical findings for study patients.

\begin{tabular}{|c|c|c|c|c|c|c|c|}
\hline Case no. & HMB-45 & S-100 & NSE & CD10 & MART-1 & Vimentin & Melan-A \\
\hline 1. & ++ & ++ & NR & NR & ++ & NR & NR \\
\hline 2. & ++ & ++ & NR & NR & NR & ++ & NR \\
\hline 3. & + & + & NR & NR & + & NR & NR \\
\hline 4. & + & + & NR & NR & + & NR & NR \\
\hline 5. & + & + & NR & NR & NR & NR & NR \\
\hline 6. & ++ & NR & NR & NR & ++ & NR & NR \\
\hline 7. & - & ++ & + & NR & - & NR & - \\
\hline 8. & - & NR & NR & NR & NR & NR & NR \\
\hline 9. & + & + & NR & NR & + & NR & NR \\
\hline 10. & $+($ in foci) & + & NR & NR & + (in foci) & NR & NR \\
\hline 11. & ++ & NR & NR & NR & ++ & NR & ++ \\
\hline 12. & + & NR & NR & NR & + & NR & NR \\
\hline 13. & ++ & ++ & ++ & ++ & - & NR & NR \\
\hline 14. & + & + & NR & NR & NR & NR & + \\
\hline 15. & - & ++ & NR & NR & - & NR & NR \\
\hline 16. & + & NR & NR & NR & + & NR & NR \\
\hline 17. & $+($ in foci) & + & NR & NR & NR & NR & NR \\
\hline 18. & + & - & NR & NR & NR & + & NR \\
\hline 19. & + & + & NR & NR & NR & NR & + \\
\hline 20. & + & NR & NR & NR & NR & NR & NR \\
\hline 21. & + & + & NR & NR & NR & NR & NR \\
\hline 22. & + & NR & NR & NR & NR & NR & NR \\
\hline 23. & - & NR & NR & NR & + (in foci) & NR & NR \\
\hline 24. & + & + & NR & NR & NR & NR & NR \\
\hline 25. & - & + & NR & NR & NR & + & + \\
\hline 26. & + & + & NR & NR & NR & NR & NR \\
\hline 27. & + & + & NR & NR & NR & NR & NR \\
\hline 28. & + & NR & NR & NR & NR & NR & NR \\
\hline
\end{tabular}

NR: Not reported; HMB-45: human melanoma black-45; NSE: neuron-specific enolase; CD10: cluster of differentiation 10; MART-1: melanoma antigen recognized by T-cells 1 .

with widespread disease, a laparoscopic exploration with left adrenalectomy, an open adrenalectomy, a splenectomy after rupture of a disease-affected spleen and a trans-anal rectal tumor debulking. Hartman's procedure and omentectomy were also performed.

Following surgery, two patients developed early intestinal obstruction and one more developed late obstruction; all three patients underwent reoperation. Thirty-day mortality was $3.6 \%$ (one patient).

In all cases, the histopathology reports were consistent with MM. The median follow-up was 11 months (range $=0.5-79$ months). The disease recurred mainly in the peritoneum (39.3\%), brain $(17.9 \%)$, and lung (10.7\%). Multifocal recurrence occurred in eight cases (28.6\%), mainly simultaneously involving the brain and the peritoneum. The median disease-free survival was 7.5 months (range $=0-75$ months). One- and 2-year overall survival was $35.7 \%$ and $10.7 \%$, respectively. Table II details these results for each patient.

Human melanoma black-45 (HMB-45) stain was positive in 23 cases (82\%); in five cases, the staining was strong and in two staining was only focal. StainingS-100 protein was positive in 18 cases $(28.6 \%$ ); in five of these cases, staining was strong. melanoma antigen recognized by T-cells 1 (MART-1) stain was positive in 10 cases (35.7\%). Results for cluster of differentiation 10 (CD10), vimentin and melanA staining were sporadically reported, therefore they were inconclusive. Infiltration of the lymph nodes by MM was positive in 11 cases (39.3\%) and negative in four (14.3\%). Table III details these results for each patient.

\section{Discussion}

Despite recent progress in combating the most common forms of cancer, MM still carries a very poor prognosis.

Geographical distribution of MM amongst the European countries is rather heterogenic, with high melanoma rates been demonstrated in Scandinavia and lower rates in the Mediterranean basin, with 15 and seven cases per 100,000 people per year, respectively (7). Geographic latitude plays a significant role in the incidence of melanoma even within 
the same nation sharing a common genetic pool, thus Norwegians inhabiting the north of their country present a 2- to 2.5-fold increase of melanoma incidence compared to those living in south Norway. Exposure to sunlight is also linked to the development of melanoma (8).

At autopsy, approximately $50-60 \%$ of patients with $\mathrm{MM}$ present with asymptomatic GI disease $(9,10)$. During the course of their disease, fewer than $4 \%$ of all patients with stage IV MM are diagnosed with GI metastases and the primary site of disease cannot be identified in up to $9 \%$ of all GI melanoma cases. In general, GI MM is difficult to diagnose and it is frequently encountered as an intraoperative finding of advanced disease with a subsequently poor prognosis (9). Immunohistochemistry is an important tool in diagnosing melanoma. The most common diagnostic markers include: S-100, HMB-45 and melan-A/MART-1 (11). Melanomas are usually negative for expression of cytokeratins and positive for vimentin, but in a number of cases they may demonstrate variable staining for anti-cytokeratin (CAM 5.2), carcinoembryonic antigen (CEA), epithelial membrane antigen (EMA), alpha-1-anticymotrypsin, and CD68 (12). S-100 protein is a sensitive ( $>90 \%$ ) but not equally specific marker for melanoma and is expressed in a nuclear pattern, although cytoplasmic staining can also occur. Positive immunoreactivity for HMB-45, melan A, or tyrosinase are much more specific markers for melanoma and melanocytes. HMB-45 shows cytoplasmic and weak nuclear staining (13). It is less sensitive, but more specific than $\mathrm{S}-100$; this marker is negative in desmoplastic melanoma. Melan-A/MART-1 is a sensitive marker, but also stains steroid-producing cells in the ovaries, testes, and the adrenal cortex. Tyrosinase is also sensitive, it stains peripheral nerve sheath and neuroendocrine tumors. NSE is a nonspecific marker. In our cases, S-100 and HMB-45 seemed to confirm their important diagnostic role. MART-1 staining was also positive in a number of cases. The rest of the markers showed inconclusive results. Expression of HMB-45 along with S-100 or MELAN-A confirms the diagnosis of melanoma.

Melanoma is a disease that is characterized by great morphological diversity and poses a diagnostic challenge to the pathologist. As mentioned above, symptoms and signs may widely vary, from asymptomatic to vague pain and weight loss, to the dramatic symptoms of an acute abdomen or bleeding. Grave presentation is not typical for GI MM. Clinical presentation of intestinal perforation or obstruction lead to emergency surgery and although this is rarely reported (14-16), the majority of our patients presented with either obstruction or bleeding. Intra-operative findings also vary and adhesions, tumors, diverticulosis and acute ischemia of the small bowel are some of the most common causes for explorative laparotomy (9).

Approximately $20 \%$ of all melanoma-affected patients will ultimately develop distant metastases and the prognosis remains poor despite existing or novel treatment modalities. Chemotherapy, interferon-alpha- or cytokines-based therapies, radiotherapy, immune checkpoint inhibitors and biochemotherapy have failed in improving the overall survival of patients with MM $(17,18)$. Vaccines have also been tested on patients with stage IV disease, but outcomes are also poor (18).

Surgery for stage IV melanoma is debatable and no clear consensus has been reached. Elective surgery seems to improve the quality of life of patients with MM, but overall survival is not affected equally (9). In a large study comparing surgery with no surgery for MM, it was reported that those patients who underwent surgery (metastasectomy) demonstrated higher median and 5-year overall survival rates compared to those who were ineligible for surgery (19). This study also concluded that surgery was an independent predictor of survival. These findings were further supported by more recent data (10). In our study, disease-free and overall survival were 7.5 (range=0-75) months and 11 (range=1-79) months, respectively. These numbers are lower than those reported in the literature, but it should be noted that all patients underwent surgery after presenting with an abdominal emergency, often meaning that the disease load was significant and prognosis without surgery could be poorer.

MM of the spleen and the adrenal glands is less frequent. Spontaneous splenic rupture usually occurs due to infectious emboli of the spleen, a pathology that was described in one of our patients. The adrenals were affected by the disease in two of our patients. Complete adrenal resection and adjuvant treatment have been advocated in such cases; as performed in these two patients $(20,21)$. Rare reports of MM to other organs, such as the stomach, colon, rectum and anus, have been also reported in literature and were also present in our group of patients (22-24).

\section{Conclusion}

In order to deliver the best of care to patients with stage IV melanoma, a rigorous and holistic approach needs to be provided by a team of expert surgeons and oncologists. Metastatic disease should be suspected in all patients with a previous history of cutaneous melanoma who present with abdominal symptoms or signs. Novel therapeutic modalities and agents show promising results in the treatment of stage IV melanoma, but their real-life benefit to the patient has yet to be proven (25-27).

Although curative surgery for stage IV melanoma remains debatable, emergency surgery for patients presenting with abdominal symptoms appears to improve survival rates and improve prognosis. Novel systemic therapies combined with surgical resection are currently being studied, with promising preliminary results. 


\section{References}

1 Amin MB, Greene FL, Edge SB, Compton CC, Gershenwald JE, Brookland RK, Meyer L, Gress DM, Byrd DR and Winchester DP: The Eighth Edition AJCC Cancer Staging Manual: Continuing to build a bridge from a population-based to a more "personalized" approach to cancer staging. CA Cancer J Clin 67: 93-99, 2017.

2 Amin MB, Edge SB, Greene FL, Byrd DR, Brookland RK, Washington MK, Gershenwald JE, Compton CC, Hess KR, Sullivan DC, Jessup JM, Brierley JD, Gaspar LE, Schilsky RL, Balch CM, Winchester DP, Asare EA, Madera M, Gress DM and Meyer LR: AJCC cancer staging manual. 8th ed. Chicago, Springer, 2017.

3 Leung AM, Hari DM and Morton DL: Surgery for distant melanoma metastasis. Cancer J 18: 176-184, 2012.

4 Hoag JR, Hegde U, Zweifler R, Berwick M and Swede H: Competing risks survival of older patients with metastatic cutaneous melanoma: a SEER population-based study. Melanoma Res 26: 505-512, 2016.

5 Cañueto J and Román-Curto C: Novel Additions to the AJCC's New Staging Systems for Skin Cancer. Actas Dermosifiliogr 108: 818-826, 2017.

6 Shenoy $\mathrm{S}$ and Cassim R: Metastatic melanoma to the gastrointestinal tract: role of surgery as palliative treatment. W V Med J 109: 30-33, 2013.

7 Maio M, Ascierto P, Testori A, Ridolfi R, Bajetta E, Queirolo P, Guida M, Romanini A, Chiarion-Sileni V, Pigozzo J, Di Giacomo AM, Calandriello M, Didoni G, van Baardewijk M, Konto C and Lucioni C: The cost of unresectable stage III or stage IV melanoma in Italy. J Exp Clin Cancer Res 31: 91, 2012.

8 Cicarma E, Juzeniene A, Porojnicu AC, Bruland ØS and Moan $\mathrm{J}$ : Latitude gradient for melanoma incidence by anatomic site and gender in Norway 1966-2007. J Photochem Photobiol B 101: 174-178, 2010.

9 Mantas D, Tsaparas P, Charalampoudis P, Gogas H and Kouraklis G: Emergency surgery for metastatic melanoma. Int J Surg Oncol 2014: 987170, 2014.

10 Rastrelli M, Tropea S, Pigozzo J, Bezzon E, Campana LG, Stramare R, Alaibac M and Rossi CR: Melanoma m1: diagnosis and therapy. In Vivo 28: 273-285, 2014.

11 Gaynor R, Herschman HR, Irie R, Jones P, Morton D and Cochran A: S100 protein: a marker for human malignant melanomas? Lancet 1: 869-871, 1981.

12 Miettinen M and Franssila K: Immunohistochemical spectrum of malignant melanoma: the the common presence of keratins. Lab Invest 61: 623-628, 1989.

13 Rothberg BE, Moeder CB, Kluger H, Halaban R, Elder DE, Murphy GF, Lazar A, Prieto V, Duncan LM and Rimm DL: Nuclear to non-nuclear PMEL17/GP100 expression (HMB45 staining) as a discriminator between benign and malignant melanocytic lesions. Mod Pathol 21: 1121-1129, 2008.

14 Catena F, Ansaloni L, Gazzotti F, Gagliardi S, Di Saverio S, De Cataldis A and Taffurelli M: Small bowel tumours in emergency surgery: specificity of clinical presentation. ANZ J Surg 75: 997999, 2005 .
15 Szynglarewicz B, Ekiert M, Forgacz J, Halon A, Skalik R and Matkowski R: The role of surgery in the treatment of colorectal metastases from primary skin melanoma. Colorectal Dis 14: e305-e311, 2012.

16 Shpitz B, Kaufman Z, Gildor A and Dinbar A: Metastatic malignant melanoma of the small bowel as a surgical emergency. Harefuah 119: 364-365, 1990.

17 Bedane C, Leccia MT, Sassolas B, Bregman B and Lebbé C; French investigators of the MELODY study: Treatment patterns and outcomes in patients with advanced melanoma in France. Curr Med Res Opin 29: 1297-1305, 2013.

18 Erdei $\mathrm{E}$ and Torres SM: A new understanding in the epidemiology of melanoma. Expert Rev Anticancer Ther 10: 1811-1823, 2010.

19 Wasif N, Bagaria SP, Ray P and Morton DL: Does metastasectomy improve survival in patients with stage IV melanoma? A cancer registry analysis of outcomes. J Surg Oncol 104: 111-115, 2011.

20 Wantz M, Antonicelli F, Derancourt C, Bernard P, Avril MF and Grange F: Long-term survival and spontaneous tumor regression in stage IV melanoma: possible role of adrenalectomy and massive tumor antigen release. Ann Dermatol Venereol 137: 464-467, 2010.

21 Flaherty DC, Deutsch GB, D Kirchoff D, Lee J, Huynh KT, Lee DY, Foshag LJ, Bilchik AJ and Faries MB: Adrenalectomy for metastatic melanoma: current role in the age of nonsurgical treatments. Am Surg 81: 1005-1009, 2015.

22 Patelis N, Marselos P, Sotiropoulou G, Georgiou S and Kominea A: Rectal melanoma. Hellenic J Surg 84: 198-202, 2012.

23 Namikawa $\mathrm{T}$ and Hanazaki K: Clinicopathological features and treatment outcomes of metastatic tumors in the stomach. Surg Today 44: 1392-1399, 2014.

24 Mourra N, Jouret-Mourin A, Lazure T, Audard V, Albiges L, Malbois M, Bouzourene H and Duvillard P: Metastatic tumors to the colon and rectum: a multi-institutional study. Arch Pathol Lab Med 136: 1397-1401, 2012.

25 Fox MC, Lao CD, Schwartz JL, Frohm ML, Bichakjian CK and Johnson TM: Management options for metastatic melanoma in the era of novel therapies: a primer for the practicing dermatologist: part II: Management of stage IV disease. J Am Acad Dermatol 68: e1-e13, 2013.

26 Amaral T, Tampouri I and Garbe C: How to use neoadjuvant medical treatment to maximize surgery in melanoma. Expert Rev Anticancer Ther 18: 121-130, 2018.

27 Garmpis N, Damaskos C, Garmpi A, Dimitroulis D, Spartalis E, Margonis GA, Schizas D, Deskou I, Doula C, Magkouti E, Andreatos N, Antoniou EA, Nonni A, Kontzoglou K and Mantas D: Targeting histone deacetylases in malignant melanoma: a future therapeutic agent or just great expectations? Anticancer Res 37: 5355-5362, 2017. 